\title{
GENESIS DE LA PRIMACÍA DEL PENSAMIENTO CALCULADOR: HEIDEGGER Y LA REPRESENTACIÓN
}

\author{
Alejandra Buchholtz F.*
}

En su crítica a la Modernidad, Heidegger parece decir que el hombre ha conseguido instalarse, gracias a las ciencias, sobre la naturaleza y, por gentileza de la técnica, sobre sí mismo. Esta situación sería posible porque el hombre ya no piensa el mundo, sino que lo calcula a modo de representación. Este artículo explora cómo la representación se transformó en el fundamento metafísico de la Época Moderna y de qué manera la ciencia moderna y la metafísica cartesiana influyeron en la gestación de este nuevo modo de "pensar".

Palabras clave: Heidegger, representación, ciencia moderna, lo matemático, Descartes.

\section{GENESIS OF THE PRIMACY OF CALCULATIVE THINKING: HEIDEGGER AND THE REPRESENTATION}

In his criticism of Modernity, Heidegger seems to affirm that man has been able to install himself, thanks to the sciences, above nature and, due to the kindness of technique, above man himself. This situation would be possible because man no longer thinks the world, but calculates it in the manner of representation. This article explores how representation became the metaphysical foundation of the Modern Era and in which manner modern sciences and Cartesian metaphysics influenced the gestation of this new manner of "thinking".

Keywords: Heidegger, representation, modern science, the mathematic, Descartes.

\footnotetext{
* Magíster en Filosofía, Universidad Adolfo Ibáñez, Santiago, Chile. Correo electrónico: alebuchholtz@gmail.com
} 
El SEMestre de invierno de 1951, HeIDEgGer inicia su curso titulado ¿Qué significa pensar? con las siguientes palabras: "Lo que más merece pensarse en nuestro tiempo problemático es el hecho que no pensamos" . Dos páginas más adelante, manifiesta la distancia que existiría entre "el pensar" y uno de los fenómenos característicos de la Edad Moderna, la ciencia, y lo hace mediante la categórica afirmación: "la ciencia no piensa"2. Años antes, en el texto La época de la imagen del mundo, correspondiente a una conferencia impartida en 1938, el filósofo hace un diagnóstico de la época moderna identificando la investigación como la esencia de la ciencia, explicando, a su vez, que la esencia de la investigación consistiría en "que el propio conocer, como proceder anticipador, se instala en un ámbito de lo ente, en la naturaleza o en la historia"3. Este proceder anticipador que se impone sobre lo ente por medio del proyecto, del rigor y de su respectivo método, llevaría al hombre a la objetivación de lo ente. Heidegger concibe tal proceder como un "representar":

...representar quiere decir traer ante si eso que está delante en tanto que algo situado frente a nosotros, referirlo a sí mismo, al que se lo representa [el sujeto] y, en esta relación consigo, obligarlo a retornar a sí como ámbito que impone las normas... ${ }^{4}$.

-"representar"- la fuerza originaria de su nombre: poner ante sí y traer hacia sí. Gracias a esto, lo ente llega a la estabilidad como objeto y sólo así recibe el sello del ser 5 .

En este "representar", el hombre, erigido como sujeto, dispone de lo ente y de su verdad en cuanto que lo convierte en objeto. A través del método, que todo lo mide, controla y predice, la ciencia moderna determina de antemano lo que son las cosas, de tal manera que los objetos son, en cuanto objetos representados. La representación sería, así, la objetivación de lo ente. Al respecto, Heidegger sostiene:

1 Heidegger, M., ¿Qué significa pensar?, Trotta, Madrid, 2005, p. 17.

2 Ibidem, p. 19. Sin embargo, en el mismo texto, páginas después, Heidegger suaviza su juicio señalando que las ciencias piensan en "su propia forma especial". Más tarde, en 1969, en una entrevista realizada por Richard Wisser a Heiddeger a sus ochenta años, el filósofo explica que con la frase "la ciencia no piensa" (1951) quiso significar: "la ciencia no se mueve en la dimensión de la filosofía; pero, sin saberlo, necesita de esta dimensión".

3 Heidegger, M., Caminos del Bosque, traducción de Helena Cortés y Arturo Leyte, Alianza, Madrid, 2000, texto La Época de la Imagen del mundo, p. 65. En adelante, esta obra se citará como La Época de la Imagen del mundo.

4 Heidegger, M., La Época de la Imagen del mundo, op. cit., p. 75.

5 Ibidem, p. 76. 
El conocimiento, como investigación, le pide cuentas a lo ente acerca de cómo y hasta qué punto está a disposición de la representación. La investigación dispone de lo ente cuando consigue calcularlo por adelantado en su futuro transcurso...En el cálculo anticipatorio casi se instaura la naturaleza, en el cálculo histórico a posteriori casi la historia. Naturaleza e historia se convierten en objeto de la representación explicativa... Sólo aquello que se convierte de esta manera en objeto "es", vale como algo que es. La ciencia sólo llega a ser investigación desde el momento en que se busca al ser de lo ente en dicha objetividad ${ }^{6}$.

Este pasaje describe la forma como procede la investigación y recalca el vínculo entre representación y objetivación. El análisis de la investigación lleva a Heidegger a identificar a la representación como el fundamento metafísico de la modernidad, diagnóstico que revela una nueva relación entre el hombre y el mundo, donde el hombre "pensaría" el mundo de un modo particular. Así, el representar se convierte en el pensamiento de vía única y la representación ya no sería un mero concepto, sino la forma como el hombre se ha instalado a sí mismo en la realidad. De acuerdo a ésta, el ente "es" en la medida en que se convierte en objeto de la representación del hombre, quien, a su vez, hace posible esta objetivación del ente al erigirse como subjectum. Como se verá, el desarrollo del método científico basado en lo matemático y, con ello, el advenimiento de la posición fundamental cartesiana, convierte al hombre en sujeto: "se convierte en aquel ente sobre el que se fundamenta todo ente en lo tocante a su modo de ser y su verdad...el hombre se convierte en centro de referencia de lo ente como tal"". Esta relación que se establece entre hombre y mundo, supone una realidad dual, en la cual, por una parte está el sujeto y, por otra, el objeto, dualidad que tendría al hombre representando y no pensando.

Cómo se fue gestando la representación o aquello que más tarde Heidegger llama "pensamiento calculador" es el tema de este artículo y se examinará complementando las ideas de proyecto y rigor, método y empresa, planteadas en La época de la imagen del mundo, con las impresiones heideggerianas acerca de la génesis de la ciencia natural moderna en La pregunta por la cosa (1935-1936). En este último libro, Heidegger explica cómo la ciencia natural moderna, principalmente las propuestas físico-matemáticas de Galileo y Newton, junto con la certeza fundamental de Descartes, confabulan para que la representación llegue a ser el fundamento metafísico de la Época Moderna.

\section{Análisis de las nociones que constituyen la esencia de la ciencia moderna y la transforman en investigación: el proyecto y el rigor, el método y la empresa}

\section{a) La investigación: proyecto y rigor}

Para el filósofo alemán lo relevante y decisivo de la investigación acontece en el mo-

Heidegger, M., La Época de la imagen del Mundo, op. cit., p. 72. 
mento en que, como "proceder anticipador", interviene el ámbito del ser al asegurarse previamente la apertura de un sector donde poder moverse.

¿De qué manera la investigación y su proceder anticipador aseguran su sector de objetos dentro del ámbito del ser? Según nuestro autor, la investigación se vincula al sector abierto por medio de "el proyecto" y "el rigor". Esto sucede de la siguiente manera: el proyecto determina anticipadamente un rasgo fundamental a proyectar, por ejemplo, en la naturaleza; luego, este proyecto define el modo en que el conocer, como proceder anticipador, debe vincularse al sector abierto. Esta forma de vincularse con lo abierto determina el rigor de la investigación. De esta manera, se podría decir que la investigación por medio del proyecto y el rigor "fuerza" la apertura de un sector que, sin embargo, para desilusión de la ciencia, continúa oculto.

Para aclarar la forma en que la esencia de la ciencia moderna se vincula con el mundo, Heidegger propone el análisis de la física matemática, la más antigua y al mismo tiempo más normativa de las ciencias modernas. La física moderna se denominaría matemática porque sería una aplicación más de las matemáticas. La relación entre matemática e investigación moderna es estudiada por Heidegger en La pregunta por la cosa, donde trata de mostrar cómo el camino que sigue la metafísica occidental provoca una crítica de la razón pura y el significado que tendría ésta. Estas reflexiones permiten entender el nexo entre investigación científica y matemática porque Heidegger, en su explicación de la génesis de La crítica a la razón pura de Kant, examina el origen de lo que denomina "la moderna ciencia natural matemática". La subtitulación utilizada a continuación es similar a la que Heidegger presenta en su texto.

\section{i. La moderna ciencia natural matemática}

Al igual que en La época de la imagen del mundo, pero con mayor profundidad, en este apartado Heidegger trata el papel decisivo que tiene el carácter matemático de la ciencia natural moderna en el modo de pensar moderno y, por tanto, en la forma de la metafísica que le es subyacente. En este capítulo el autor intenta mostrar que el pensamiento moderno pretende relacionarse con el ser de la cosa a la manera como opera la moderna ciencia natural matemática.

Heidegger propone la comprensión de la ciencia natural moderna en comparación con la forma de hacer ciencia de los griegos. Según él, "la posición fundamental [del pensamiento] frente a las cosas y al ente en general"8 es clave para la establecer una distinción entre la ciencia natural griega y la moderna. Heidegger explica la transformación de la ciencia en base a dos fundamentos:

7 Ibidem, p. 73. 
1. -sobre la experiencia del trabajo, es decir, la dirección y el modo del dominio y la utilización del ente". 2. -sobre la metafisica, es decir, sobre el proyecto del saber fundamental del ser, a partir del cual se construye el ente en cuanto inteligible ${ }^{9}$.

Estos fundamentos le permiten sostener que la diferencia entre ambas surge de la manera en que los hechos -y con ellos los experimentos- son comprendidos y los conceptos aplicados. La ciencia moderna se caracterizaría, así, por tres elementos. Primero, es una ciencia de hechos. Segundo, estos hechos suponen una investigación experimental. Finalmente, lo más característico de la ciencia moderna es que tanto hechos como experimentos son abordados de antemano desde el cálculo y la medición. Subyacente a estas tres características, de manera normativa, está el rasgo fundamental del pensamiento moderno, esto es, "que la nueva exigencia de saber es exigencia matemática"10.

\section{ii. Descripción de lo matemático}

Heidegger intenta aclarar la noción de lo matemático recurriendo al significado originario de la palabra, que provendría del griego: "lo que se puede aprender, y por eso también lo que se puede enseñar"11. Si bien el autor explica lo matemático como aquello que se puede tanto aprender como enseñar, este estudio se concentrará en lo matemático como un aprender. En este sentido, lo matemático se refiere a las cosas, pero desde la siguiente perspectiva. Si se entiende lo matemático como un aprender, y este aprender como una forma del tomar y tomar significa: "apropiarse de algún modo de una cosa y disponer de ella"12, se desprende que lo matemático se refiere a las cosas en cuanto que éstas son aprendibles, es decir, aprendidas a conocer de acuerdo al modo matemático.

Así, lo matemático conoce de las cosas, aquello "de"13 las cosas que en realidad ya conoce, como explica Heidegger, "por consiguiente no es algo [lo que conocemos] que extraemos de las cosas sino algo que, en cierto modo, llevamos con nosotros mismos"14. Si lo matemático es lo que se conoce antes de referirse a la cosa, se advierte que lo numérico es algo matemático, aspecto que se puede apreciar en el siguiente ejemplo de Heidegger:

Vemos tres sillas y decimos: son tres. Lo que es "tres" no nos lo dicen ni las tres sillas, ni los tres gatos, ni cualquiera otras tres cosas. Más bien, podemos contar solamente tres cosas como tres, si ya conocemos el tres ${ }^{15}$.

8 Heidegger, M., La Pregunta por la cosa, Memphis, México, 2002, p. 67. En adelante se citará como La pregunta por la cosa.

9 Heidegger, M., La Pregunta por la cosa, op. cit., p. 67.

10 Ibidem, p. 70.

11 Ibidem, p. 71.

12 Heidegger, M.., La Pregunta por la cosa, op. cit., p. 72.

13 La preposición "de" va entre comillas porque lo que lo matemático conoce no es algo "de" la cosa.

14 Heidegger, M., La Pregunta por la cosa, op. cit., p. 75.

15 Idem 
Cuando conocemos tres cosas, por tanto, solo conocemos de ellas lo ternario, conocimiento que estaba en el pensamiento antes de relacionarse con las cosas. ¿Dónde está el número tres? En nuestra mente. Los números son lo más inmediato que conocemos de las cosas, sin extraer ese conocimiento de ellas. En la ciencia moderna el aprender consiste en tomar en conocimiento algo que de alguna manera ya se posee. Para Heidegger, en la Modernidad ese conocimiento es el verdadero aprender, se toma conocimiento de lo numérico haciendo abstracción de las cosas. De ahí que el número, por su naturaleza, es comprendido como algo matemático. Luego, lo matemático tendría un doble significado: sería lo que es aprendible antes de vincularse a la cosa y también el mismo modo del aprender y del proceder, por lo cual, lo matemático debe conocer su fundamento. "Lo matemático es aquella posición fundamental (presupuesto básico) en la cual nos pro-ponemos las cosas en aquel modo en que ya nos son dadas y deben ser dadas"16 [como axioma concebido desde la mente].

\section{iii. Carácter matemático de la ciencia natural moderna:}

\section{a. Ley del movimiento de los cuerpos de Newton}

Heidegger explica cómo lo matemático se transforma en el rasgo fundamental de la ciencia moderna haciendo referencia a Newton y su obra Philosophia naturalis principia mathematica (1686-1687), dado que aquella encontraría su principal fundamentación en la ley del movimiento de los cuerpos de Newton, formulada en los siguientes términos:

Todo cuerpo persevera en su estado de quietud o en el movimiento uniformemente rectilineo, en tanto en cuanto no esté forzado por fuerzas impresas a cambiar aquel estado ${ }^{17}$.

Esta ley ${ }^{18}$ "de inercia", al ser considerada como ley fundamental (axioma), generó una revolución en el pensamiento y derivó en una nueva concepción sobre la naturaleza. Si bien esta ley fundamental era desconocida antes de Newton, tenía ciertos precedentes. Según Heidegger, Galileo la aplicó, pero en forma no explícita, y Descartes, en su obra Principia Philosophiae, la incluyó e incluso trató de fundamentarla metafísicamente ${ }^{19}$.

Vale la pena detenerse en algunas expresiones de esta ley debido a que dan cuenta del modo en que la ciencia moderna enfrenta a la naturaleza. Las expresiones "todo cuerpo" y "uniformemente" no tendrían ningún sentido al referirse a la naturaleza en el mundo griego. En cambio, en la Modernidad ellas determinan el curso de la ciencia natural.

16 Heidegger, M., La Pregunta por la cosa, op. cit., p. 79.

17 Heidegger, M., La Pregunta por la cosa, op. cit., p. 79.

18 De acuerdo a Heidegger, la ley, en la ciencia moderna, es una regla que fija lo que hay de variable en los hechos, es decir, determina una constante necesaria en las transformaciones que acontecen en el transcurso de ciertos hechos.

19 En su Tratado del mundo Descartes también se ocupa del movimiento de los cuerpos y la inercia. 


\section{b. Comparación entre la ciencia natural griega y moderna, centrada en el pensamiento de Aristóteles y Newton}

Si bien - según Heidegger - la ciencia física griega se guiaba por las representaciones y principios planteados por Aristóteles y por ciertas influencias del mundo de las ideas de Platón, esto no significa que los conceptos elaborados por Aristóteles carecieran de referencia a las cosas. Por el contrario, señala Heidegger, Aristóteles se ocupó de que el pensar fuera siempre "un decir, que corresponda a lo que se muestre en el mismo ente" 20 , agregando que: "los griegos caracterizan las cosas...como algo que surge de sí mismo [de la cosa], y algo que se produce, que se elabora"21, recalcando que en Aristóteles el concepto sí se refería a la cosa.

Así, en lo que respecta al procedimiento científico, tanto Aristóteles como Newton tendrían una actitud fundamental similar en cuanto a que los conceptos y principios se obtienen desde los fenómenos, tal como lo señalan las palabras del propio Newton:

En la ciencia experimental las proposiciones extraídas de los fenómenos por inducción deben ser tenidas como verdades exactas, o como verdades muy aproximadas, si no se le oponen supuestos contrarios, hasta que surgen otros fenómenos, por los cuales o se los formula de una manera más exacta o se los puede someter a excepciones ${ }^{22}$.

Sin embargo, sí existe diferencia entre el quehacer científico de griegos y modernos. La diferencia entre Aristóteles y Newton residiría esencialmente en lo que "se apresa como fenómeno y la manera como se lo interpreta" ${ }^{23}$. Con esta aclaración, Heidegger busca mostrar que la gran diferencia está en qué entiende cada uno por fenómeno y de qué forma se interpreta lo que cada cual determina como fenómeno, es decir, de acuerdo a la posición fundamental del pensamiento - la manera en que Aristóteles y Newton se ubican frente a las cosas y al ente en general.

A partir de la cita de Newton también se puede derivar otra diferencia esencial entre la ciencia griega y la moderna: Newton en su proposición utiliza más de una vez la palabra "exactas", lo cual revela la pretensión de exactitud de las ciencias modernas. Heidegger destaca que la ciencia griega no tenía aspiraciones de verdades exactas: "La ciencia griega nunca fue exacta, porque según su esencia era imposible que lo fuera y tampoco necesitaba serlo"24. En este pasaje, el autor distingue entre esencia de la ciencia griega y esencia de la ciencia moderna, distinción que tiene su fundamento en la concepción de lo ente y la interpretación de verdad que se tiene en cada uno de estos momentos, confirmando que para Heidegger lo decisivo radica en la posición fundamental del pensamiento.

\footnotetext{
Heidegger, M., La Pregunta por la cosa, op. cit., p. 81.

Ibidem, p. 82.

Heidegger, M., La Pregunta por la cosa, op. cit., p. 81.

Ibidem, p. 82.

24 HeidegGer, M., La Época de la Imagen del mundo, op. cit., p. 64.
} 


\section{c. Doctrina del movimiento en Aristóteles y Newton}

La comparación entre la doctrina del movimiento de Aristóteles y de Newton contribuye a la comprensión del cómo la ciencia física moderna se convirtió en matemática.

Para Heidegger las dos doctrinas coinciden en que los cuerpos de la naturaleza están en movimiento y quietud, considerando quietud como un modo del movimiento. En suma, la diferencia entre ambas teorías residiría en la relación entre los cuerpos y el movimiento, y el lugar que les correspondería.

Para Aristóteles los cuerpos se separan en terrestres y celestes y cada cual se movería y ocuparía un lugar de acuerdo a su propia naturaleza. Por lo tanto, según su naturaleza, los cuerpos ígneos tienen su lugar arriba y se mueven de manera circular y los cuerpos terrestres tienen su lugar abajo y se mueven en forma rectilínea. De esta manera, el modo de moverse de los distintos cuerpos y la relación a su lugar dependen de la naturaleza del cuerpo. En palabras de Heidegger: "según la concepción aristotélica la causa, en los movimientos naturales, está en la naturaleza del cuerpo mismo, en su esencia, es decir, en su ser más propio" ${ }^{25}$. El filósofo luego muestra cómo a este pensamiento le correspondería un principio de la Escolástica posterior: "el modo del movimiento sigue el modo de ser"26.

Newton comienza la ley de inercia con: "Todo cuerpo....", borrando para siempre la distinción entre cuerpos celestes y terrestres. Todos los cuerpos son concebidos, en esencia, iguales. Como consecuencia, la superioridad del movimiento circular frente al movimiento rectilíneo no es un hecho; por el contrario, es el movimiento rectilíneo el que da la norma (es impuesto a todos los cuerpos): "Todo cuerpo persevera en su estado de quietud o en el movimiento uniformemente rectilíneo..."; adicionalmente, se le agrega la noción de uniformidad ${ }^{27}$. Esto significa asimismo una alteración en el concepto de lugar. El lugar ya no es la ubicación que le corresponde a un cuerpo según su naturaleza, sino que éste se relativiza de acuerdo a su relación con otras situaciones. Así, el movimiento de un cuerpo está supuesto y no se discute. Lo que se cuestiona es la causa de un cambio en el movimiento uniformemente rectilíneo de un cuerpo, por ejemplo, un movimiento circular. De esta manera, si el movimiento de un cuerpo se define como uniformemente rectilíneo y el lugar que ocupa es relativo a ciertas situaciones, el movimiento consiste sólo en cambio y relación de situaciones, como lo sería la distancia de lugares. En consecuencia, el movimiento se determina por mediciones de distancia, por magnitudes de movimientos.

Así, la naturaleza ya no es el principio interno causante de los movimientos de los cuerpos. Esto implica que la naturaleza, en general, deja de ser cuestionada como posee-

25 Heidegger, M., La Pregunta por la cosa, op. cit., p. 86.

26 Idem.

27 El concepto de uniformidad adquiere en la modernidad un valor antes no visto. Tanto Heidegger como su discípula Hannah Arendt critican y sospechan del afán de uniformar de lo Moderno. 
dora de un orden interno. Esta nueva doctrina del movimiento transforma, según Heidegger, el concepto de naturaleza. La naturaleza comienza a ser ordenada, por ejemplo, por ámbitos como el tiempo y el espacio, los cuales, pueden ser medidos y calculados ${ }^{28}$. De esta manera, Heidegger vincula el primer principio del movimiento de Newton con todos los cambios acontecidos en el modo de preguntar por la naturaleza, ya que la nueva posición fundamental presente en el axioma de Newton es matemática.

\section{iv. Relación entre lo matemático y la formulación del principio fundamental de la ciencia moderna enunciado por Newton}

Para explicar la presencia de lo matemático en el primer principio fundamental de la ciencia moderna Heidegger recurre al antecedente de éste. Según él, lo matemático estaría ya presente en el principio que formuló Galileo para fundamentar la caída de los cuerpos:

Concibo un cuerpo arrojado sobre un plano horizontal, excluido todo obstáculo, resultará entonces, de lo que en otra parte se dice en forma más detallada, que el movimiento del cuerpo sería uniforme y perpetuo si el plano se extendiera en el infinito"..."concibo en mi mente algo movible totalmente abandonado a si mismo ${ }^{29}$.

En este principio, según Heidegger, Galileo anticipa el movimiento:

Lo preconcebido por Galileo con respecto al movimiento fue la determinación de que el movimiento de todo cuerpo es uniforme y rectilíneo, si se excluye todo obstáculo, pero que también se altera uniformemente al sufrir la influencia de una fuerza constante ${ }^{30}$.

Antes de realizar el experimento de la caída de los cuerpos, Galileo formuló un principio, el cual determina, a modo de ley, el comportamiento de los cuerpos cuando caen. Con las palabras "concibo en mi mente" Galileo comienza por dar cabida a lo matemático en las ciencias naturales. En su mente Galileo concibe un cuerpo que, según Heidegger, no existe, pero el físico italiano anticipadamente determina el movimiento que seguirá dicho cuerpo. De esta manera, la ciencia moderna que pretende fundamentarse en la experiencia se fundamenta en un principio supremo. Aquello que se concibe en la mente, que habla de una cosa que no existe y que, a la vez, pretende fundamentar la cosa, reposa en lo matemático. En este sentido, las ciencias naturales modernas son matemáticas porque actúan determinando la cosa sin haber obtenido esa determinación desde la cosa. Más aún, la cosa misma ha sido determinada anticipadamente desde la mente.

28 Juan de Dios Vial Larraín describe esta situación de la siguiente manera: "El comportamiento recíproco de los cuerpos, sean astros, cometas, el mar o las manzanas, se define por el producto de las masas y la inversa del cuadrado de la distancia", en el libro La Vía de la Verdad. El discurso de la metafísica, Ediciones Universidad Católica de Chile, Santiago, 2006, p. 23.

29 Heidegger, M., La Pregunta por la cosa, op. cit., p. 90.

30 Idem. 
Heidegger interpreta el pensamiento de Galileo y Newton, diciendo:

...se concibe de antemano aquello que debe ser uniformemente determinante para todo cuerpo como tal, es decir, toda corporeidad. Todos los cuerpos son iguales. Ningún movimiento tiene preferencia. Todo lugar es igual a otro; todo punto temporal es igual a otro...Todas las determinaciones se proyectan en un plano horizontal, según el cual el proceso natural no es otra cosa que la determinación espacio-temporal del movimiento de unidades de masa. Ese plano de la naturaleza delimita al mismo tiempo su ámbito como homogéneo ${ }^{31}$.

La física moderna se llama "matemática" porque aplica el pensamiento matemático en un sentido eminente. Este aspecto es precisado por Heidegger, en el texto La época de la imagen del mundo, cuando define la física del siguiente modo:

La física es el conocimiento de la naturaleza en general y particularmente el conocimiento de lo que tiene un carácter corpóreo y material en su movimiento, pues esto corpóreo se muestra de modo inmediato y penetra todo lo natural aunque sea de distintas maneras. Pues bien, si ahora la física se configura expresamente bajo una forma matemática, esto significa que, gracias a ella y por mor de ella, algo se constituye por adelantado y de modo señalado como lo ya conocido. Esta decisión afecta nada menos que al proyecto de lo que a partir de ese momento deberá ser naturaleza en aras del conocimiento de la naturaleza que se persigue: la cohesión de movimientos, cerrada en sí misma, de puntos de masa que se encuentran en una relación espacio-temporal ${ }^{32}$.

Este análisis muestra cómo la ley de inercia formulada por Newton, antes sugerida por Galileo y Descartes, permitió que "el universo se poblara de cuerpos todos de la misma índole, sujetos a un solo tipo de movimiento regido por la ley de inercia, y susceptiblemente de ser matemáticamente conocido"33. Este cambio en el modo de conocer la naturaleza se fue gestando desde el momento en que Galileo afirmó que el universo estaba escrito en el lenguaje de la geometría, para luego instalarse cuando Newton explica los fenómenos por medio del cálculo infinitesimal.

\section{v. Lo matemático como proyecto}

Hacia el final del capítulo La moderna ciencia natural matemática y la génesis de una crítica de la razón pura, Heidegger completa la determinación de la esencia de lo matemático describiéndolo a modo de "proyecto", en los siguientes términos:

i) La cosidad procede como una mente que concibe abriendo un ámbito en el que se muestran las cosas. ii) Lo axiomático establece de antemano el principio-fundamento de

31 Heidegger, M., La Pregunta por la cosa, op. cit., p. 91.

32 Heidegger, M., La Época de la Imagen del mundo, op. cit., p. 66.

33 Vial Larraín, Juan de Dios, La Vía de la Verdad, El discurso de la metafísica, op. cit., p. 21. 
la cosa que se expresa como axioma. iii) La proyección matemática al constituirse como axioma, pre-aprehende la cosa al modo que se prefigura como esquema fundamental la estructura de cada cosa y de sus relaciones con toda otra cosa. iv) La "naturaleza" es delimitada por el mismo esquema fundamental del axioma. Por lo tanto, "naturaleza" es aquello definido por el proyecto, desapareciendo ésta como principio interno causante de los movimientos de los cuerpos:

Naturaleza es ahora el ámbito configurado en el proyecto axiomático de la conexión de movimientos uniformes espacio-temporales. Los cuerpos sólo pueden ser cuerpos en tanto están incluidos y entretejidos en ese ámbito ${ }^{34}$.

v) El vínculo proyecto-objeto es descrito por Heidegger de la siguiente manera: "Los cuerpos naturales son tal como se muestran en el ámbito del proyecto" ${ }^{35}$. Esto significa que el cómo se muestran los cuerpos está ya prefigurado por el proyecto, por lo cual, la experiencia de la naturaleza se convierte en experimento (en sentido moderno). Mediante el experimento la naturaleza responde de acuerdo a las condiciones formuladas de antemano por el proyecto. vi) Lo matemático, como proyecto, presenta exigencia numérica dado que el proyecto busca imponer uniformidad a todo cuerpo (a partir de relaciones de espacio, tiempo y movimiento), por lo tanto, requiere de la medida continuamente uniforme, esto es, de la medición numérica.

Así, para Heidegger, lo matemático es "un tomar conocimiento, que se da desde sí mismo aquello que toma, y se lo da como aquello que ya tiene" ${ }^{\prime 36}$.

\section{b) Método y empresa en la investigación}

Si la ciencia se convierte en investigación de la naturaleza por medio del proyecto y del aseguramiento del mismo en el rigor del proceder anticipador, la naturaleza "es" en cuanto cuestionable y determinable por magnitudes espacio-temporales de movimiento con carácter de exactitud. La aspiración de alcanzar exactitud matemática sólo es posible mientras proyecto y rigor se conviertan en lo que son en el método. El método, que es tal, al modo matemático, determina el segundo carácter esencial para la investigación.

\section{i. El método}

Respecto al método, Heidegger busca revelar que lo esencial del procedimiento del método es que consigue que la naturaleza (e incluso la historia) y, específicamente, el sector proyectado, se vuelva objetivo. Para lograr la objetivación de todo lo ente, el método, a

34 Heidegger, M., La Pregunta por la cosa, op. cit., p. 92.

35 Idem.

36 Heidegger, M, La Pregunta por la cosa, op. cit., p. 91. 
través del proceder anticipador, debe representar aquello que se da como una constante en el ámbito variable de los hechos de la naturaleza. De esta manera, el método consiste en fijar una constante considerando el movimiento como tal. Para que esto suceda, el método recurre tanto a la regla como a la ley. La regla es "la fijación de los hechos y la constancia de su variación como tal" y la ley es "lo constante de la transformación en la necesidad de su transcurso" 37 . Así, la investigación moderna pretende aclarar la naturaleza a través de la imposición de reglas y leyes. Sin embargo, para llevar a cabo la aclaración de un sector de objetos la investigación necesita del experimento.

Por medio del experimento, la investigación fundamenta algo desconocido por medio de algo conocido y, a la vez, garantiza eso conocido por medio de algo desconocido. Cuando Heidegger se refiere a lo conocido está haciendo referencia a la regla y a la ley. Luego, el experimento moderno es posible en la medida que las ciencias naturales modernas se han convertido en investigación al modo matemático: "la física moderna puede ser experimental gracias a que es esencialmente una física matemática"38.

El método de la investigación consiste en experimentar instalando, como fundamento, de antemano, una ley. Por lo tanto, el experimento se realiza de acuerdo a lo que se dispone en una ley, que se establece como una hipótesis que se desarrolla, no en forma arbitraria, sino que a partir de un rasgo fundamental de la naturaleza. Luego, el experimento, como procedimiento, es ejecutado de acuerdo a la hipótesis con el objetivo de producir los hechos que confirman o niegan la ley. Mientras más exactamente se haya proyectado el rasgo fundamental de la naturaleza, afirma Heidegger, mayor será la posibilidad de que el experimento arroje resultados positivos, es decir, confirme la ley. El filósofo recalca que el experimento moderno utiliza un método esencialmente diferente al practicado en la Antigüedad y en la Edad Media, en cuanto que de éste se espera un "tipo de confirmación de la ley en el marco y al servicio de un proyecto exacto de la naturaleza" 39 .

Heidegger cierra su intervención con respecto al método en su texto La época de la imagen del mundo, señalando que éste pretende, tanto en las ciencias naturales modernas como en las ciencias históricas, la objetivación de lo ente al representar aquello que es constante. De esta forma, lo decisivo es que en la Modernidad el método tiene supremacía sobre lo ente, tal como lo expresa en La pregunta por la cosa: "El método no es una pieza indumentaria de la ciencia entre otras, sino la instancia fundamental a partir de la cual se determina lo que puede llegar a ser objeto y cómo puede llegar a serlo" ${ }^{\text {. }}$.

Por último, para Heidegger el método, en cuanto que debe mostrar resultados y con ellos abrirse camino para obtener nuevos resultados, no tiene el mismo modo para todas

37 Heidegger, M., La Época de la Imagen del mundo, op. cit., p. 67.

38 Idem.

39 Heidegger, M, La Época de la Imagen del mundo, op. cit., p. 68.

40 Heidegger, M, La Pregunta por la cosa, op. cit., p. 100. 
las ciencias. Toda ciencia, en cuanto que su esencia, la investigación, está fundada sobre un proyecto referido a un sector de objetos delimitados, es una ciencia particular. Esto implica que toda ciencia debe especializarse para asegurar el progreso de toda investigación y participar de un tercer proceso fundamental, la empresa.

\section{ii. La empresa}

La investigación procede como empresa en el momento en que el método se conduce de acuerdo a las posibilidades abiertas por el mismo, esto es, al regirse por sus propios resultados y luego establecer un nuevo proceder anticipador.

Es en la empresa, que aparentemente sería el cuarto paso de la investigación, “en donde por primera vez el proyecto del sector de objetos se inscribe en lo ente" ${ }^{91}$. Con esto, Heidegger intenta explicar que la forma de proceder de la investigación como empresa, es decir, todo lo referente a disposiciones que facilitan acuerdos conjuntos, planificaciones de los modos del método, comparación de resultados, regulación de las fuerzas de trabajo, es la forma como la investigación asegura la primacía del método por encima de lo ente. En consecuencia, el carácter de empresa de la investigación permite que las ciencias alcancen, según sus propias palabras: "la mutua pertenencia y la unidad que les corresponde" ${ }^{\prime 42}$. Según Heidegger, la ciencia particular consigue unidad con otras ciencias particulares en su calidad de empresa y gracias al investigador que trabaja en proyectos de investigación, que tiene más que ver con un técnico que con un erudito. Sin embargo, en la práctica, en la universidad se ve la tendencia de las ciencias a separarse y especializarse, sin consumarse la particular unidad interna, antes descrita, que se supone debiera surgir del carácter de empresa de la investigación.

Así, la investigación se convierte en tal gracias a las constantes exigencias recíprocas que se dan entre el proyecto, el rigor, el método y la empresa, las que permiten representar aquello que se da como una constante en el mundo y obtener la objetivación de lo ente.

Heidegger resume esta situación de la siguiente manera:

La ciencia moderna se basa y al mismo tiempo se especializa en proyectar determinados sectores de objetos. Estos proyectos se despliegan en los correspondientes métodos asegurados gracias al rigor. El método correspondiente en cada caso se organiza en la empresa ${ }^{43}$.

41 Heidegger, M., La Época de la Imagen del mundo, op. cit., p. 70.

42 Heidegger, M., La Época de la Imagen del mundo, op. cit., p. 70.

43 Ibidem, p. 71. 


\section{Relación del pensamiento de Descartes con el establecimiento de lo matemático como rasgo fundamental del pensar moderno y con el origen del fundamento metafísico de la modernidad, esto es, con el concepto de representación.}

Si bien para Heidegger lo matemático es el rasgo fundamental del pensamiento moderno, la conformación del pensamiento metafísico de dicha era, es consecuencia del modo momentáneo y concreto de la existencia histórica. Según él, junto al dominio de lo matemático como modo de conocer, hay una nueva posición fundamental de la existencia. Esta nueva posición fundamental de la existencia se caracteriza por dejar atrás la verdad como revelación divina y dar lugar a la búsqueda de la verdad por medio de la razón.

Si las certezas se alcanzan haciendo debido uso de la razón, lo matemático cumpliría con los requisitos para fundar la ciencia moderna, toda vez que el proyecto matemático acarrea: liberación (respecto de la doctrina de la Escolástica) y, como consecuencia de ello, una nueva noción de libertad concebida como autonomía; autodependencia (subordinación a sus propios principios); y autofundamentación (lo matemático se fundamenta a sí mismo con axiomas autoevidentes). Esto significaría que la esencia del proyecto matemático tiene propiedad para la transformación y autofundación del saber como tal. El filósofo de Friburgo explica así las pretensiones del rasgo fundamental del pensar moderno: "lo matemático tiende por sí mismo a poner su propia esencia como fundamento de sí mismo, y con ello de todo saber"44. Como se verá, este proyecto matemático, que depende de los principios exigidos por sí mismo, se traslada, incluso, hasta la filosofía.

Para comprender cómo lo matemático se pudo convertir en medida de la filosofía, nuestro autor considera indispensable examinar la propuesta metafísica de Descartes (1556-1650). Como muchos otros, Heidegger considera que la filosofía moderna comienza con Descartes ${ }^{45}$, sosteniendo incluso que sería su responsabilidad la configuración filosófica de lo matemático como rasgo fundamental de la existencia moderna.

Para analizar el pensamiento cartesiano es indispensable referirse a la aspiración categórica de Descartes de erradicar la incertidumbre e investigar la verdad por medio de un método y un fundamento racionalmente asentado que permita alcanzar certezas evidentes y absolutas en el ámbito del conocimiento.

En el diálogo que emprende con Descartes, tanto En la pregunta por la cosa como En la época de la imagen del mundo, Heidegger examina principalmente dos textos cartesianos. Por un lado, estudia una obra temprana e inacabada de Descartes titulada Regulae ad directionem ingenii (Reglas para la dirección del espiritu) escrita alrededor de 1628 y

\footnotetext{
44 Heidegger, M. La Pregunta por la cosa, op. cit., p. 96.

45 Algunos pensadores, como Risieri Frondizi, -traductor de Descartes-, estiman que el año de publicación del Discurso del método, 1637, podría escogerse como la fecha simbólica en que comienza la filosofía estrictamente moderna.
} 
publicada en forma póstuma en 1701, que pareciera constituir, por sobre El Discurso del Método, la fuente principal para estudiar la noción cartesiana de método. Posiblemente por tal motivo, en referencia a este texto, el filósofo desarrolla el vínculo del pensamiento cartesiano con la exigencia matemática como carácter fundamental del pensamiento moderno. Por otro lado, complementa su diagnóstico con respecto a la modernidad con el análisis de la propuesta metafísica de Descartes presente en sus Meditaciones Metafísicas, obra en la cual su autor manifiestaría que la nueva acometida a la realidad debía emprenderse, por un lado, desde una reflexión de lo matemático ${ }^{46}$ en su significación fundamental $\mathrm{y}$, por otro lado, necesariamente, desde una meditación metafísica que examine la posición fundamental del pensar en referencia a todo lo ente.

Así la tarea de la filosofía tendría una doble dimensión: metodológica y metafísica. La relación entre ambas se daría de la siguiente forma: "el método debe enseñarnos a usar la razón que la metafísica nos enseña a conocer" ${ }^{\text {" }}$. Con respecto a este vínculo método-metafísica, Heidegger estima que la actitud fundamental filosófica de Descartes avanza, a la par, en dirección a una fundamentación de lo matemático desde una reflexión metafísica.

La necesidad de que la reflexión matemática se convierta en reflexión metafísica se explica cuando se comprende que lo matemático pretende transformarse en norma del pensar. Heidegger explica esta relación entre lo matemático y lo metafísico cuando se refiere a lo matemático del siguiente modo: "significa que lo matemático quiere [requiere] fundamentarse a sí mismo en el sentido de su propia exigencia interna; quiere destacarse él mismo, expresamente, como norma de todo pensar y formular reglas que derivan de ello"48.

Esta doble intención se manifestará también en Reglas para la dirección del espíritu. Heidegger explica este título como: "principios y directivas en los cuales lo matemático se subordina a sí mismo a su esencia; una fundamentación de lo matemático para que se convierta en su totalidad en una norma para el espíritu investigador"49. Para el filósofo, este título expresa tanto el rasgo de auto-fundamentación de lo matemático como una posición fundamental frente a lo ente, en cuanto que el investigador se relacionaría desde lo matemático, a modo de norma, con la totalidad de lo ente. En esta formulación de Descartes estaría presente la idea de las matemáticas como una ciencia universal ${ }^{50}$, esto es, como "la ciencia única y normativa, que relaciona y configura todo" ${ }^{51}$. Sin embargo, Descartes no termina, ni publica esta obra, con lo cual, quizás no estuvo convencido del planteamiento de las ideas expresadas en este texto.

46 Lo matemático venía perfilándose como rasgo fundamental del pensar desde hacía un siglo.

47 Rábade Romeo, S., El Racionalismo. Descartes y Espinosa, Trotta, Madrid, 2006, p. 70. En adelante este texto se citará como El Racionalismo. Descartes y Espinosa.

48 Heidegger, M., La Pregunta por la cosa, op. cit., p. 99.

49 Idem.

50 En este aspecto, Rábade coincide que Descartes, al igual que Espinoza, Leibniz, Locke o Hume, aprecia las matemáticas como la scientia universalis.

51 Heidegger, M., La Pregunta por la cosa, op. cit., p. 99. 
Con respecto a las matemáticas, es indispensable precisar que si bien Descartes manifiesta en sus obras metafísicas que ciencias como la Aritmética y la Geometría "contienen algo de cierto y de indudable" ${ }^{52}$, reconoce que en las matemáticas no está la certeza primera: "gustaba sobre todo, de las matemáticas, por la certeza y evidencia de sus razones; pero me extrañaba que, siendo sus conocimientos tan firmes y sólidos, no se hubiese construido sobre ellos nada más elevado" 53 . Para él, las matemáticas no constituían el principio primero, es más, Descartes recurre a las matemáticas solo como ejemplo para establecer su método, es decir, como modelo metodológico ${ }^{54}$.

Con respecto al método, concebido como necesidad primera, lo decisivo es la forma en que la valoración de lo matemático, reflejada en el método, afectó la controversia con la metafísica tradicional, y la manera en que ésta determinó la figura de la filosofía moderna.

Heidegger resulta indispensable cuestionar la posibilidad de las matemáticas de transformarse en fundamento de todo el saber. Por lo tanto, plantea, a partir de lo expuesto por Descartes en Reglas para la dirección del espíritu, que si lo matemático fundamenta y configura la totalidad del saber, debe formular ciertos axiomas eminentes, que deben ser:

1. Absolutamente primeros, evidentes en sí, por sí, es decir absolutamente ciertos...2. Los axiomas supremos deben...fijar de antemano con respecto al ente en su totalidad qué es existente y qué significa ser, desde dónde y cómo se determina la cosidad de las cosas ${ }^{55}$.

Por ello, para la posición fundamental matemática no puede haber cosas dadas previamente. Por lo tanto, si hasta ahora la proposición ha sido propuesta como algo que en cierto modo se presentó por sí misma y, que contiene y retiene lo que las cosas son, esto significa que la proposición existe también al igual que las cosas, es decir, la proposición sería el continente existente del ser. De esta manera, Heidegger piensa que para que la proposición no sea arbitraria debe estar colocada sobre su fundamento, esto significa que la propia proposición debe ser en sí misma un principio, esto es, el principio absoluto. De acuerdo a esto, lo matemático exige encontrar una proposición inicial, en la cual aquello sobre lo que ella dice algo, el subjectum ${ }^{56}$, no se toma de otra parte que no sea ella misma. Por lo tanto, esta proposición originaria debe constituirse en lo subyacente como tal. De esta manera, siendo la posición de la proposición de lo matemático el subjectum, esto es, el fundamento absolutamente cierto e indudable, todo saber anterior debe ser cuestionado.

52 Descartes, R., Meditaciones Metafísicas, Alianza, Madrid 2009, p. 84. En adelante se citará como Meditaciones Metafísicas.

53 Descartes, R., Discurso del Método, traducción de Risieri Frondizi, Alianza, Madrid, 2011, p. 94. En adelante se citará como Discurso del Método.

54 Rábade destaca como uno de los caracteres básicos de la modernidad filosófica a la "irrenunciable preocupación metodológica".

55 Idem.

56 Heidegger entiende la palabra subjectum de acuerdo a una traducción del griego, como "lo que yace ante nosotros, y que, como fundamento reúne todo sobre sí. Cfr. HeidegGer, M., La época de la imagen del mundo, op. cit., pp. $72-73$. 
En esta parte del texto La pregunta por la cosa, el filósofo profundiza en la reflexión matemática de Descartes en relación con la meditación metafísica cartesiana, tomando como fuente, por lo que se desprende, el texto Meditaciones Metafísicas.

Como se mencionó anteriormente, para Descartes no basta con colocar lo matemático como fundamento absoluto de todo saber de la naturaleza, sino que debe encontrar el principio primero y supremo para el ser del ente en general. Este principio, absolutamente matemático, no podría aceptar nada recibido de antemano. Así, si la proposición se entiende en el sentido del pensar enunciativo: "la proposición sólo se tiene a sí misma como lo que puede ser puesto" 57 . Por ello, el enunciar y el pensar sólo alcanzan certeza absoluta en la medida que se construye sobre un punto de partida indubitado: ese punto de partida solo puede ser el "yo pienso". Esto significa que cuando se hace una enunciación sobre cualquier cosa, para que sea evidente en sí misma, ella debe realizarse desde el "yo pienso". Según el filósofo, el pensar de Descartes se formulará necesariamente de la siguiente forma: "Pensar es siempre un "yo pienso", ego cogito" 58 . Esta forma de concebirse a sí mismo del pensamiento tendría como fundamento lo matemático: "Recién cuando el pensar se piensa a sí mismo será absolutamente matemático, esto es, un tomar conocimiento de aquello que ya tenemos"59. Así, Descartes alcanza la primera certeza en el momento que el pensamiento se piensa a sí mismo siguiendo el método matemático.

En El Discurso del Método, por primera vez, a partir de su duda metódica, Descartes da con la certeza primera en la cual el pensar se piensa a sí mismo:

Pero advertí en seguida que aun queriendo pensar, de este modo, que todo es falso, era necesario que yo que lo pensaba, fuese alguna cosa. Y al advertir que esta verdadpienso, luego soy-era tan firme y segura que las suposiciones más extravagantes de los escépticos no eran capaces de conmoverla, juzgué que podía aceptarla sin escrúpulos como el primer principio de la filosofía que buscaba ${ }^{60}$.

Es importante precisar qué entiende Descartes por pensar. En el texto Los principios de la Filosofía, lo explica así:

Con el nombre de pensamiento entiendo todo lo que ocurre en nosotros estando conscientes, hasta donde hay en nosotros conciencia de esos hechos. De manera que no sólo comprender, querer, imaginar, sino también sentir significa aqui lo mismo que pensar $^{61}$.

57 Heidegger, M., La Pregunta por la cosa, op. cit., p. 102.

58 Idem.

59 Idem.

60 Descartes, R., Discurso del Método, op. cit., p. 123.

61 Descartes, R., Los Principios de la Filosofía, Losada, Buenos Aires, 1997, Primera Parte, IX, p. 9. 
En Las Meditaciones Metafísicas, formula el primer principio del siguiente modo:

De suerte que, después de haber pensado y de haber cuidadosamente examinado todas las cosas, es preciso, por último, concluir constantemente que esta proposición: yo soy, yo existo, es necesariamente verdadera, siempre que la pronuncio o que la concibo en mi entendimiento $^{62}$.

Con respecto a esta verdad completamente evidente, Descartes considera necesario precisar el yo soy:

Pero, ¿Qué soy yo? Una cosa que piensa. Y ¿qué es una cosa que piensa? Es un cosa que duda, que entiende, que concibe, que afirma, que niega, que quiere, que no quiere, que imagina y que siente t3. $^{6}$.

Descartes define el yo como una cosa pensante, que duda, quiere, siente..., a la cual denomina, res cogitans. Por otro lado, identifica a aquello que representa el mundo exterior de los cuerpos como res extensa. La res extensa está determinada, ante todo, por su extensión y movimiento (además por la forma, el tamaño, la cantidad, el lugar y el tiempo). Esta aclaración permite comprender cómo todas estas "cualidades primarias" permiten que la naturaleza sea concebida cuantitativa y matemáticamente desde la razón.

Según Armando Roa, la Modernidad empieza con la separación dual cartesiana del alma y el cuerpo ${ }^{64}$. Roa interpreta esta concepción dualista de Descartes de la siguiente manera: "tal separación deja al cuerpo como una simple máquina susceptible de ser conocida y manejada por la físico-matemática; por tal vía, el alma creadora de dicha físicomatemática llegará a tener algún día, según se espera, el manejo total del cuerpo"65. Descartes, junto con establecer la separación entre pensamiento y cuerpos extensos, entrega el dominio de lo corporal a lo espiritual al proponer al "yo soy, yo existo" como primer fundamento. Esta noción dual del mundo, consecuencia del primer principio cartesiano, tendrá como consecuencia la distinción entre sujeto y objeto.

Heidegger analiza el surgimiento de la primera certeza de Descartes del siguiente modo. El principio primero y supremo para el ser del ente en general, formulado en el Discurso del Método como "pienso luego soy" y enunciado luego en las Meditaciones Metafísicas como "yo soy, yo existo", se comprende como un "yo soy”. Así, "cogito ergo sum" no sería una deducción: "El sum no es consecuencia del pensar, sino por el contrario

62 Descartes, R, Meditaciones Metafísicas, op. cit., p. 89.

63 Idem., p. 93.

64 HeidegGer sostiene en varios de sus textos que la concepción dual del mundo, que ha determinado el curso de la metafísica occidental, tiene su origen en el pensamiento de Platón. "La posición metafísica fundamental de Descartes está soportada por la metafísica platónica-aristotélica y se mueve, a pesar del nuevo comienzo, dentro del mismo planteamiento: ¿qué es lo ente?”. Heidegger, M., La Época de la Imagen del mundo, op. cit., p. 80.

65 RoA, A., Modernidad y Posmodernidad, Andrés Bello, Santiago, 1995, p. 29. 
es su fundamento"66. Por lo tanto, el "pienso luego soy", debe entenderse como un "yo pongo", porque en la esencia de la posición que el hombre asume frente al mundo desde el principio fundamental "yo soy" estaría la proposición: "yo pongo".

Heidegger interpreta el primer principio de Descartes como un "yo pongo" considerando que esta proposición no se dirige a algo dado de antemano, sino que sólo se da a sí misma lo que en ella hay. El "yo soy" cartesiano significaría un "yo pongo" puesto que, al anteponer el yo en su proposición, Descartes hace del yo, como lo ponente, aquello pro-puesto como lo ya dado y, por lo tanto, se convierte en el ente mismo. A partir del "yo soy", el ser del ente es determinado como la certeza del poner. Incluso, quizá, desde el "yo soy", podría decirse: "yo soy, las cosas son". Así, Heidegger explica la proposición cartesiana de la siguiente forma:

Yo pongo, yo soy el que pone y piensa. Esta proposición tiene la particularidad de que en ella primero se pone aquello acerca de lo cual ella enuncia, el subjectum. Lo que ella pone es en este caso el "yo"; el yo es el subjectum del principio primero. Por eso el yo es lo subyacente (Zugrundeliegendes) eminente -subjectum- el subjectum de la posición general. Por eso es que desde entonces se toma el yo preferentemente como el subjectum, como "sujeto" ${ }^{67}$.

Heidegger intenta mostrar, por un lado, que sólo fue posible que el yo alcanzara estatus de subjectum debido al dominio esencial y radical de lo matemático y de lo axiomático (el yo caracteriza "lo ya dado propia y previamente" ${ }^{\prime 68}$ ). Por otro lado, pretende confirmar cómo, a partir de la proposición fundamental "yo soy", el hombre se convierte en el sujeto que fundamenta la totalidad de lo ente. Para él, es importante aclarar que la conversión del yo de Descartes en sujeto preeminente no tiene un sentido subjetivo. Esto se explica comprendiendo que el yo cartesiano tiene su fundamento en un "yo pienso" que ha sido concebido matemáticamente, es decir, la supremacía del yo no significaría que la subjetividad $^{69}$ humana se haya establecido como centro del pensar. Por el contrario, el yo como "yo pienso" pasa a ser el fundamento de toda certeza y verdad; es decir, el yo se convierte en la fundamentación del saber total. Asimismo, desde el "yo pienso" la determinación de lo racional adquiere una preeminencia evidente porque el pensar es el acto esencial de la razón. La razón se erige como principio supremo:

... ahora la razón, como el "yo pienso", se coloca expresamente en el principio supremo, como hilo conductor y tribunal de todas las determinaciones del ser $^{70}$.

66 Heidegger, M., La Pregunta por la cosa, op. cit., p. 103.

67 Idem.

68 Idem.

69 Para Heidegger, considerar lo planteado por Descartes como una posición subjetivista correspondería a una interpretación "vulgar" del pensamiento cartesiano, opinión que manifiesta de la siguiente manera: "Esta historia de Descartes, que vino, dudó, y se convirtió en un subjetivista fundando la teoría del conocimiento, nos da la imagen corriente. A lo sumo, es una mala historia, pero no una historia en la que se haga visibles el movimiento del ser". Heidegger, M., La Pregunta por la cosa, op. cit., p. 98.

70 Heidegger, M., La Pregunta por la cosa, op. cit., p. 105. 
Como se ha explicado, según Heidegger el yo cartesiano es un "yo pongo" que determina el ser del ente. Desde esta posición, en la cual, "el ser del ente se determina desde el "yo soy" como la certeza del poner" 71 , el hombre surge como sujeto y transforma al resto del mundo en objetos conocibles matemáticamente. Así, para él, la propuesta de Descartes permite que el hombre sea quien fundamente toda la realidad:

Hasta Descartes cada cosa presente por sí se tomaba como "sujeto"; pero ahora el "yo" se convierte en sujeto preeminente, en aquel en relación al cual las cosas restantes se determinan como tales. Puesto que ellas -matemáticamente obtienen su cosidad por la relación fundante con el principio supremo y su "sujeto" (yo), son esencialmente tales que están relacionadas al "sujeto" como lo otro, contrapuestas a él como objectum. Las cosas mismas se convierten en "objetos" 72 .

\section{Balance: transmutación de la noción de subjectum}

En su análisis del pensamiento cartesiano, Heidegger ratifica al hombre como el subjectum, como aquello que fundamenta matemáticamente la totalidad de lo ente, e introduce el objectum en oposición al subjectum. Esto significa que por objectum se entienden las cosas, en tanto que, éstas se transforman en objetos en oposición al sujeto. Las nociones de subjectum y objectum se transforman. El subjectum ya no es aquello que "yace frente al hombre y que reúne todo sobre sí"73 sino que, es el hombre el subjectum en cuanto fundamenta todo lo ente "en lo tocante a su modo de ser y su verdad"74. Por otro lado, el término objectum también sufre un cambio de significación. De acuerdo a Heidegger, hasta antes del advenimiento de la modernidad, "objectum significó lo contra-yecto en el mero representarse" 75 o, como aparece en otra traducción del texto La pregunta por la

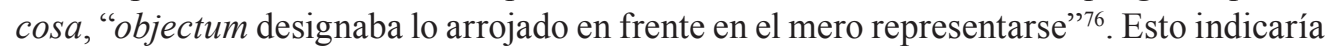
que aquello representado por el objectum era algo meramente "subjetivo" (de acuerdo al uso lingüístico actual). Por ejemplo: me represento un río de brillantes. Lo representado, al modo de un objectum en el lenguaje de la Edad Media, sería en la Época Moderna algo subjetivo porque no existe un río de brillantes. Así, lo que era considerado el objectum era algo que, no existía "objetivamente" (de acuerdo a su actual uso lingüístico).

Para Heidegger, esta inversión en los significados de los términos subjectum y objectum tiene relación con una transformación metafísica:

...es un cambio transmutador de la existencia (Dasein), es decir, del claro (Lichtung) del ser del ente, en razón del dominio de lo matemático. Es un tramo del camino de la historia

71 Ibidem, p. 102

72 Ibidem, p. 103.

73 Heidegger, M., La Época de la Imagen del mundo, op. cit., pp. 72-73.

74 Ibidem, p. 73.

75 Heidegger, M., La Pregunta por la cosa, op. cit., p. 104.

76 Heidegger, M., La Pregunta por la cosa, Editorial Palamedes, 1987, p. 136. 
auténtica, necesariamente oculto para el ojo habitual, historia que es siempre la de la patencia del ser- o no es nada ${ }^{77}$.

A partir de esta situación, en opinión de Heidegger, la razón humana, desde la posición que le concede el "yo pienso" cartesiano, se ubica como el centro de referencia desde donde todo ente recibe todas sus determinaciones, en cuanto a su ser y a su verdad (a pesar de que para Heidegger el ser se mantendría oculto para este sujeto).

En consecuencia, la primera certeza, asentada matemáticamente y establecida por Descartes, acarrea consigo una nueva relación entre el hombre y el mundo. El siguiente esquema representa el vínculo que existiría entre el hombre y el mundo:

\section{$\mathrm{S} / \mathrm{O}$}

En este esquema la $\mathrm{S}$ representa al hombre como sujeto, que si bien se encuentra separado del mundo, representado por la $\mathrm{O}$, determina al mundo desde el momento en que lo transforma en objeto. La nueva situación podría describirse así: el hombre desde el axioma fundamental "yo pienso" se convierte en el sujeto que decide de antemano aquello que es objeto y las determinaciones que a éste le corresponden.

Heidegger reacciona a esta explícita división sujeto/objeto. Para él, esta relación entre el hombre y el mundo, en la cual, el hombre imprime el ser al mundo, revela que el hombre estaría vinculándose al mundo al modo de una representación. En opinión de Heidegger, quien concibe que el pensamiento sólo es pensamiento en cuanto atiende al ser, el hombre moderno se encontraría constantemente representando y no pensando. Desde esta meditación surge un diagnóstico desgarrador respecto de la experiencia del pensar y, por lo tanto, del hombre en la Época Moderna: El hombre se ha erigido como el gran sujeto representador, el ente se ha convertido en objeto de representación, la verdad es entendida como certeza de la representación y el mundo se ha transformado en imagen.

\section{Conclusión}

Heidegger dedica la segunda etapa de su vida intelectual a estudiar la experiencia del pensar, como una forma de perseverar en su más íntimo anhelo: la superación de la metafísica y su olvido del ser. Si bien en obras como ¿Qué significa pensar? explora el camino hacia el aprendizaje del pensamiento reflexivo, sus obras anteriores resultan imprescindibles como crítica a la existencia histórica moderna bajo el dominio de la ciencia y la técnica. De diversos modos, incluso desde la poesía, Heidegger parece decir que sólo cuando el hombre moderno reconozca el alcance y límite de la posición fundamental de su existencia en la era técnica, caracterizada por la imposición de axiomas sobre las cosas, podrá soñar con habitar el mundo.

77 Heidegger, M., La Pregunta por la cosa, op. cit., p. 104. 
Los textos estudiados en este artículo revisten valor en cuanto revisan cómo la conformación de la ciencia moderna y el pensamiento cartesiano llevaron a su consolidación la escisión iniciada por Platón. Así, el estudio de la génesis del fundamento metafísico de la época moderna -la representación o pensamiento calculador-permite al hombre la comprensión de su situación actual y posibilita un movimiento.

Heidegger no se resigna al "hombre" que constantemente calcula el mundo, al hombre que concibe el mundo como una suma de fenómenos medibles en magnitudes de tiempo y espacio, los cuales no serían más que enunciados que habitan en la razón. El carácter matemático de las ciencias modernas exactas y el principio primero de Descartes "pienso luego soy", entendido por Heidegger como un "yo pongo", influyeron para que el pensamiento calculador dejara de ser exclusividad de la ciencia y la técnica y se transformara en la única vía de pensamiento. El proceder anticipador de la ciencia, junto con la intensificación de la conciencia, han llevado al hombre a instalarse de tal manera que el mundo resulta determinado desde elaboraciones de la mente, separándolo de la naturaleza.

Para el hombre que es capaz de reconocer esta "huida del pensar" y que vive "tiempos de penuria", el filósofo propone el pensar reflexivo o meditativo, que sería el pensar propiamente tal, en cuanto que da la posibilidad al hombre de ser hombre mientras deja al mundo ser mundo en una relación que supera la división entre sujeto y objeto. El hombre pensará la naturaleza cuando renuncie a su posición de sujeto y aprenda a estar-en-elmundo atendiendo a lo abierto, al claro, al ser.

En una alocución pronunciada en 1955, Heidegger insiste en lo inquietante que resulta la primacía del pensamiento calculador. Sin embargo, también ahí, reconoce y justifica como necesarios los dos tipos de pensar: el pensar calculador y el pensar reflexivo. Hacia el final llama a los hombres a que se comprometan a reaccionar frente al poder oculto de la técnica mediante una actitud que él denomina Serenidad.*

\section{Bibliografía}

Heidegger, Martin, ¿Qué significa pensar?, Trotta, Madrid, 2005.

Alianza, Madrid, 2000.

, Caminos del bosque, traducción de Helena Cortés y Arturo Leyte, La Pregunta por la cosa, Memphis, México, 2002.

, La Pregunta por la cosa, Palamedes, Tübingen, 2009.

* Artículo recibido: 25 de octubre de 2014. Aceptado: 12 de diciembre de 2014 